\title{
Impact of Artificial Intelligence on the health protection scheme in India
}

Impact of Artificial Intelligence in India

\author{
Raul V. Rodriguez \\ Woxsen University, Hyderabad, India \\ Sanjivni Sinha \\ Citius Tech, Mumbai, India, and \\ Sakshi Tripathi \\ HDFC Bank, Mumbai, India
}

\begin{abstract}
Purpose - The purpose of the paper is to highlight the role of Artificial Intelligence (AI) in the healthcare industry through the Ayushman Bharat health protection scheme by analyzing various technologies being integrated to improve the customer service and experiences in India. The key focus lies on the understanding of the influence of $\mathrm{AI}$ in the healthcare system services, the clinical treatment, and the facilities to progress with accurate and precise health screening in India.

Design/methodology/approach - A systematic study on the emerging technologies of AI and the applications in the healthcare sector is presented in the form of a viewpoint.

Findings - AI certainly enhances experiential services; however, it cannot surpass the human touch which is an essential determinant of experiential healthcare services. AI acts as an effective complementary dimension to the future of healthcare.

Originality/value - This viewpoint discusses the applications and role of AI with the help of relevant examples. It highlights the different technologies being applied and how they will be used in the future focusing upon the Ayushman Bharat health protection scheme in India.
\end{abstract}

Keywords Healthcare, Health protection scheme, Technological change, Artificial Intelligence, India

Paper type Viewpoint

\section{Introduction}

Artificial Intelligence (AI) is defined as intelligence that is demonstrated by machines which mimic human cognitive functions (Jiang et al., 2017). Nowadays, AI is being used in several fields such as defense, healthcare, banking and more. In order to provide quality healthcare, AI will be mainly utilized to tackle structural factors such as shortage of professionals, discriminatory access to healthcare or concentration of healthcare facilities in Tier 1 and Tier 2 cities (PwC, 2018). Indian healthcare will be worth US $\$ 280$ billion by 2020; thus, it is one of India's largest professional sectors in terms of employment and revenue (India Brand Equity Foundation, 2019). Some of the growth drivers are rising income level, more diseases due to lifestyle, increasing level of health awareness and accessible insurance policies. Ayushman Bharat (which means "bless India with long healthy life") Scheme represents the largest health protection program funded by the government with a budget of US\$887.04 million for the year 2019-20. The program targets to achieve 600 million beneficiaries, and approximately

(c) Raul V. Rodriguez, Sanjivni Sinha and Sakshi Tripathi. Published in Public Administration and Policy. Published by Emerald Publishing Limited. This article is published under the Creative Commons Attribution (CCBY 4.0) license. Anyone may reproduce, distribute, translate and create derivative works of this article (for both commercial and non-commercial purposes), subject to full attribution to the original publication and authors. The full terms of this license may be seen at http://creativecommons. org/licences/by/4.0/legalcode

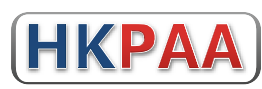



India, 2018).

\section{Artificial Intelligence in healthcare}

$\mathrm{AI}$ is a game-changer, and the healthcare sector will be able to avail several opportunities from this technological development. The areas where AI has been rapidly emerging include machines that can sense, comprehend, learn and act in order to execute administrative and clinical healthcare functions (Radick, 2017).

AI increases the scope of activities that can be done by machines such as natural language processing, chatbots, computer vision or machine learning. For example, machine learning can be deployed to understand the overwhelming healthcare data while reducing the decision processing time (Westgate, 2017). IBM's Watson is used in oncology procedures to prescribe the treatment which is more suitable for the patients (Reddy et al., 2015). There are new startups in India using AI to address challenges related to the quality delivery, automatic diagnosis, detection and screening of diseases or predictive healthcare diagnosis. Similarly, it will be conducive towards the eradication of discriminatory treatments based on social or structural backgrounds.

\section{Government initiatives}

Some factors which will be highly affected by the integration of AI in the healthcare industry are cost, quality, efficiency and reach of healthcare to the underserved areas. The major focus is on providing services to those areas where the infrastructure is not yet available or primary healthcare quality is questionable. However, a full-fledged replacement of physicians and doctors by automation is still a debatable topic which will be socially addressed in the future. On the other hand, the larger impact of AI in India is focused upon addressing issues related to economic disparity. Some of the initiatives taken by the government include the tie-up of its think tank, National Institution for Transforming India Aayog (NITI Aayog) with Google to encourage AI research, hence, contributing to the technology-empowered New India (NITI Aayog, 2018). Furthermore, NITI Aayog is working at a national level to deploy an analytical portal with the help of AI which aims to make National Health Scheme (NHS) a centralized health record system which will help in the effective management of health information with the use of big data analytics and machine learning (Dhupar, 2017).

The Indian government pursues initiatives in boosting the AI impact in healthcare to enhance the quality of health protection in the rural parts of India. Ayushman Bharat National Health Protection Scheme was an initiative to provide quality and affordable healthcare to all citizens. It is the world's largest healthcare scheme dealing with strategic management challenges such as database maintenance and quality controls. The Indian government has deployed AI, deep learning and machine learning to deal with such situation.

\section{Challenges}

There are several opportunities for AI in India, but challenges related to technology management need to be dealt with first. On the one hand, we must discuss the ethical implications AI use in healthcare and the possible options to solve such situations. AI will be falling under four main types of ethical difficulties: bias, lack of consciousness, lack of data privacy and singularity - the growth of AI intelligence beyond humans. The key framework to tackle these issues is to assure the backend programming and data implementation are sufficiently versatile among different software engineers originating from socio-cultural backgrounds along with the utilization of reinforcement learning in AI, which is the learning by trial and error in order to achieve the desired target (IBM, 2014).

An adequate progression is possible only when there is a robust legal framework in place. Some of the challenges are the institutional regulation of authority, appropriate certification 
mechanisms, infrastructure, economic investment, unanswered legal queries and information asymmetries and perceptions. Currently, India lacks a regulating body regarding AI impact in the healthcare sector to assure data security which will prevent the data from being misused (Stead, 2018). India is devoid of such mechanism which leads to expensive clinical trials, excessive time consumption and lack of standard quality certifications resulting in the unacceptability of the data (Stead, 2018). Therefore, one of the possible solutions could be to have doctors as panel members while deploying AI in the sector.

Infrastructure is a tremendous challenge in India when it comes to AI implementation as it requires adequate cloud computing, high speed internet and computing power which are yet largely unavailable in several regions of the country, leading to hosting servers abroad (Srivastava, 2018). Additionally, AI is underfunded by the public sector in India and is currently limited to just a few professional areas.

Information asymmetries and perceptions are another area of concern as doctors and coders do not have any specific standards to meet the uniformity, resulting in hesitation to adopt new software and adaptability to use them. Moreover, there is a lack of understanding about the required policies, terms and conditions to use such technology along with a large set of unanswered legal questions which will ensure responsibility in case of any error committed by the professionals involved (Gupta and Kumari, 2017). Therefore, it is of utmost importance to formally establish rules and regulations which will adhere to the situational instances and working circumstances.

\section{Applications of AI in the various healthcare segments in India}

The impact of $\mathrm{AI}$ in big data in research has been ever-growing in recent years with the development of chatbots, personalized clinical diagnosis, and a higher degree of disease predictive accuracy setting a differentiation benchmark between traditional IT and AI. Some hospitals in India have witnessed the employment of descriptive and predictive AI first-hand. For example, Manipal Group of Hospitals implemented such technological advancements in order to help doctors to provide accurate treatments to cancer patients through IBM Watson, in its oncology specialization.

Another example of AI being used in disease detection is Aravind Eye Care, where the data collected are analyzed for early detection of diseases such as diabetes, blood pressure, among others (Vadakkepat et al., 2015). In the pharmaceutical industry, AI can be helpful in enhancing the value proposition, sales automation and market differentiation. For example, Pharmarack utilizes AI within the supply chain to ensure optimization of the line (eHealth, 2019).

In the field of diagnosis, startups and MNCs are harnessing the potential of $\mathrm{AI}$ in premature disease detection and diagnosis, including Google and IBM. Alongside this, several startups are using deep learning and machine learning to detect the early symptoms and recommended personalized care actions. In the startup universe, examples are Cureskin, which focuses upon skin diseases such as scars, pimples and warts, as well as Qure.ai, which uses deep learning to enable early detection and further procedures.

Another area where AI is extremely helpful is in the counselling psychology industry. Depression is generally seen as a stigma by Indian society. Hence, AI helps providing chatbots (e.g. Wysa) which assure empathetic support and suggest when to consult human practitioners. Anonymity is a vital factor in this case which helps the patients sharing their feelings without being concerned of being judged or categorized.

Moreover, Telemedicine helps in providing quality and affordable treatment in the rural parts of India, and AI eradicates the discriminatory factor among the masses (Chandwani and Dwivedi, 2015). An example of business activity in this field is, SigTuple, an Indian startup which analyzes blood samples and generates reports without the help of pathologists. Another example is Philips Innovation Campus (PIC), which enabled healthcare to become affordable and accessible by use of AI. Philips, in partnership with Fortis Escorts Heat

\section{Impact of Artificial Intelligence in India}


Institute, Delhi, has created IntelliSpace Consultative Critical Care, which helps monitoring

different intensive care units from a central command center.

\section{Ayushman Bharat - National Health Protection Scheme}

India's population is about 1.3 billion which is just slightly less than China's population. Consequently, one of the main challenges is to provide quality healthcare to all its citizens. Though India's majority of the population $(65 \%)$ are young and under 35 years old, the remaining are mostly aging and require quality and affordable health protection system. As such, the Indian government launched one of the world's largest health schemes covering approximately 100 million underprivileged and 500 million vulnerable families by providing coverage up to Rs 50 lakhs rupees per family for secondary and tertiary care. This is the Ayushman Bharat National Health Protection Scheme (Singh, 2018), originated in 2018 under Ministry of Health and Family Welfare.

According to Lahariya (2018), some of the key features of Ayushman Bharat are:

(1) The scheme has specified coverage of Rs 5 lakhs rupees per family per year.

(2) It is transferable in nature across the country. The beneficiary can avail cashless coverage benefit from any empaneled hospitals across the country.

(3) The National Health Protection Mission is based on entitlement which is decided based on the Socio Economic and Caste Census database.

(4) Both public and private hospitals are listed from where beneficiaries can avail the scheme.

(5) In order to restrict payments for treatments, pre-defined package is made.

(6) Core governing principle of Ayushman Bharat is co-operative federalism and state flexibility.

(7) To have robust and scalable IT platform, National Health Protection Mission has partnership with NITI Aayog to have paperless, cashless transaction.

(8) AI will be implemented in order to assure higher accuracy and diagnosis performance through intelligent systems based on machine and deep learning. This will be highly beneficial for the patients.

The scheme includes Rs 5 lakhs rupees insurance per year which can be utilized not only by individual users but family members alike. In case of multiple surgery requirements, the highest package is provided followed by $50 \%$ waiver for the second time, and $25 \%$ waiver for the third time. The cashless treatment is shared between the central government and state government in the 60:40 ratio (Jha, 2018). "The beneficiaries, once verified as genuine, receive the card which can be shown to any empaneled hospital to utilize the service. Most of the critical diseases are included in the scheme, barring few like cosmetic surgery and fertility related procedures. The scheme's aim is to provide quality healthcare to all the citizens" (Keshri and Gupta, 2019, p. 65). However, there are many challenges which Ayushman Bharat faces. In the subsequent sections, the authors will detail how AI could overcome such difficulties.

\section{AI utilization in solving the Ayushman Bharat challenges}

One of the success stories of AI for Ayushman Bharat is enabling cashless transaction at multiple levels (Bakshi et al., 2018). In case of an emergency, patients can go to any hospitals and procure the treatment. In addition, they receive notifications from the recommendation system which listed the best hospitals to visit in order to diagnose their symptoms. As for the doctors, they could now acquire information about the patients via 
an online channel. This has reduced the stress level of the patients, eased the transaction system and helped in procuring the required treatment. In addition, with the help of AI, all the transactions are cashless. Ayushman Bharat is currently facing a lot of strategic management challenges like data handling of all the patients and citizens enrolled in the scheme, minimizing the cost of service, improving data security and quality of care. In order to enhance the service, the Government of India has deployed AI, machine learning and deep learning.

Below are four challenges along with respective solutions on how AI can resolve the problems.

\section{A. Minimizing the cost of services at the expense of clinical care}

In India, every year more than 60 million people, who are below the poverty line, are not able to avail healthcare due to its high cost (Angell et al., 2019). Currently, it is observed that the Indian healthcare delivery system lacks standardization and, as a result, the cost of providing the service is exorbitant. Therefore, one of the most important challenges Ayushman Bharat faces is reducing costs in order to meet the volume and quality. An alternative way in which hospitals can reduce costs is using AI in emergency cases, where lot of tests need to be done in a short period of time (Angell et al., 2019). AI not only reduces the time taken for getting the results of tests, but also decreases the doctor's time in performing those functions. As a result, the doctors can treat more patients at lower cost and standardization can be achieved.

Online platforms and gateways are required to maintain detailed information on medicines, drugs, and instruments available so that it can be accessed easily by everyone. The supply chain management is important for managing any healthcare organization (Bakshi et al.,2018). One of the applications of AI is to automatically send the inventory orders when the level of medicines reach certain a threshold, while considering the doctors' feedback about how the medicine works on the patients. In case any medicine has a negative feedback, AI would filter it out and raise an alarm so that correct intervention by doctors can be taken and medicine can be replaced, or further investigation can be directed.

There are many startups worldwide which are or will be legally stationed in medical fields to help doctors and patients in several ways.

(1) Ada: It is a machine learning chatbot which provides a platform to directly communicate with the patients to give recommendations. The application communicates with the patient about symptoms and issues which s/he is experiencing. Consequently, the chatbot provides proper feedback and offers specialist details for remote consultation (Ada, 2020).

(2) Lunit: Lunit is a medical AI software company devoted to conquering cancer and advancing medical intelligence to the next level. Lunit develops and provides novel AI-powered solutions for cancer diagnostics and therapeutics which can save time and save lives. It is utilizing deep learning and 3D visualization so that it can be used to detect the unnoticed diseases like airway cancer, colorectal cancer etc. It aims to perform with $83 \%$ to $86 \%$ accuracy (Lunit, 2020).

(3) Sense.ly: This chatbot is similar to Ada but has some different and advance services like reminder for medications, visit to the doctor as well as insurance recommendations (Sense.ly, 2020).

(4) Insilico Medicine: It was developed in 2014 and performs one of the most important tasks in the medical field - drug discovery at minimal cost and time rate. The company aims to make a better living for all human beings (Insilico, 2020).
Impact of Artificial Intelligence in India 
PAP

23,3

\section{8}

(5) PathAI (Boston): A graduate from Harvard medical school, Andy Back developed this software to help the doctors find accurate cell images, which helps them identify the patient's disease (PathAI, 2020).

(6) Aira (San Diego): It provides vision to visually impaired people. With the help of Artificial Intelligence, a smart glass helps the person seeing the world. For the blind, it can perform a simple task like reading (Aira, 2020).

(7) Julia: It helps in diagnosing diabetic retinopathy through appropriate use of deep learning. It provides accurate reports of the respective test (Anantharaman and Shah, 2016).

\section{B. Ensuring quality of care}

With the assurance of quality of care in Ayushman Bharat, the hospitalization rate of lowest $40 \%$ of the population is expected to increase from $2.45 \%$ to $2.75 \%$, which is about 17 million patients per year (National Health Authority, 2018). AI plays an important role in ensuring the quality of care provided to the increasing number of patients. With the help of Artificial Intelligence, both quality and cost will be affected, as efficient work minimizes the time period, which consequently reduces a large conglomerate of people at various places like reception, diagnosis centers, medical store and alike. Although machines play a major role in improving the quality of service, the accumulation of bacteria on those machines can lead to hospital-acquired diseases (National Health Authority, 2018). However, with the help of bacteria or infection tracker devices, which are fully automated, the infection rate can be decreased. An accurate alarming system can be devised to monitor and provide an indication when the rate of infection reaches a certain point.

\section{Quality and security of data}

Ayushman Bharat relies heavily on the development of data which have to be maintained effectively. Data privacy is another issue which needs to be addressed as it provides security to patient stakeholders, service providers and so on (National Health Authority and Quality Council of India, 2019). A proper structure of the data follows some guidelines such as collection, storage, completeness and exchange, which ensures that the data is reliable and in an accepted format. However, due to lack of proper system, one of the challenges faced by the patients includes inaccessibility of their medical records and therefore, they fail to present the necessary documentation to another consultant which causes inconvenience in case of emergencies. The second challenge is the securitization of the data, i.e., ensuring security of data and its privacy during storage, standardization and exchange among different entities (National Health Authority and Quality Council of India, 2019). Nowadays, collection of amounts, receipt, storage facility and transfer of sensitive information by service providers to respective authorities are subjected to the Information Technology Rules, 2011 which are safeguarded under the Information Technology Act, 2000. Information and data shared among hospitals, diagnostics centers and clinics need to be maintained properly.

Both challenges can be overcome through deep learning. For example, in hospitals, by utilizing various software, data and information are secured and easily accessible. Similarly, IT heads can lead and circulate information between hospitals and authorities with the help of programming languages such as R and Python.

\section{Reducing frauds}

Globally, it has been reported that US $\$ 260$ billion (5.59\%) of global healthcare spending is lost in frauds yearly (Reuters, 2010). Healthcare schemes, insurance policies and programs, which are mainly organized by the government, do not obtain consistent information from the subordinates, leading to fraudulent activities in the entire system. This results in malpractices, providers' persuasive demands, fake policyholders, fake beneficiaries and 
alike. In order to address these malpractices in Ayushman Bharat, there is a framework subjected to Anti-fraud Guidelines, 2018, that aims to detect, prevent and deter fraudulent and abusive activities in the system (Dey, 2019).

The Government of India has placed an extra layer of protection by means of AI to monitor the trend and formulating standard treatment protocols to check the irregularities caused due to over-billing or over charging, over testing, wrong beneficiary information and abuse in referral mechanism. The scheme touched almost 300,000 beneficiaries within 10 months. The Government has employed the National Health Authority (NHA) for the prevention of deception in the scheme, which continuously detects the potential scams and carries a detailed investigation before taking any measures against them (National Health Authority, 2018).

The NHA had detected anomalies in 48 hospitals across the country, out of which 31 were suspended due to the following reasons:

(1) Doctors' inconsistencies during working hours in public hospitals.

(2) Doctors in public hospitals illegally referring their patients to private hospitals by issuing referral slips for pecuniary benefits.

(3) Referring patients from private clinics to the private hospitals.

(4) Hospitals falsified information related to a clause under the scheme, which stated that a patient will be granted benefits from the scheme if $\mathrm{s} / \mathrm{he}$ is admitted in the hospital for 6 hours and more.

As a result of these irregularities, the NHA designed an IT system with the help of machine learning, which helps hospitals and diagnostics centers to access real-time information including activities such as beneficiary identification, fund flows, transaction management, claims payment, referred patients' details, etc. With the help of this system, the NHA maintains stringency in the system for tightening the noose around insurance companies, private and public hospitals and ensuring the smooth distribution of healthcare benefits to the beneficiaries. With the established parameters defining the fraudulent activities, and through the implementation of facial and voice recognition software in CCTV cameras, plausible frauds could be prevented by AI.

\section{Conclusion}

Artificial Intelligence, machine learning and deep learning bring several opportunities in all the professional arenas, but healthcare must be highlighted as one of the most prominent areas wherein AI can be helpful in managing administrative difficulties, process standardization, cost reduction, among others. The government intervention has also enhanced the AI ecosystem by providing opportunities for startups to address this new venture, while encouraging well-established technology firms to collaborate with hospitals and employ the same technology. However, challenges such as the lack of a proper regulatory body and software certification, have made doctors question the future outcomes of this implementation. Therefore, in order to increase the level of acceptance, the government needs to address the prevalent issues at the earliest possibility.

AI is being used in hospitals to detect diseases, handle pharmacy supply chain management, detect fraudulent activities, and organizing numerous administrative activities. Ayushman Bharat, one of the Indian government's flagship schemes, seeks to provide healthcare insurance to all the citizens of India. The scheme is likely to succeed as AI has and will play a major role in its smooth functioning. In order to provide quality healthcare to rural India, telemedicine has been deployed and data securitization is confirmed by using AI. Furthermore, the government has added extra layer in reducing frauds and ensuring high quality service. In a nutshell, AI will help healthcare sector in standardizing the process, reducing cost, eliminating frauds and making quality healthcare service available for all.

\section{Impact of Artificial Intelligence in India}


PAP

23,3

280

It is important to mention that AI will not be able to replace healthcare professionals permanently, instead it will enable them to increase their performance accuracy as well as the diagnosis rate. On the other hand, psychological distress and anxiety might present due to the fear of job loss. It can be reduced by slowly introducing physicians to the concept of AI implementation and providing them with an adequate time frame for adjustment.

The Ayushman Bharat scheme serves about 50,000 underprivileged citizens who can avail the benefits outside their districts and states from 2019. Similarly, the true effectiveness of the scheme should be evaluated by the end of 2022 as per the initial government plans and directives (Government of India, 2018) in an ethnographic mode and experiencing the service first-hand.

\section{References}

Ada (2020), "About us", available at: https://ada.com (accessed 7 August 2020).

Aira (2020), “About us", available at: https://aira.io (accessed 7 August 2020).

Anantharaman, R. and Shah, V. (2016), "Deep learning in Julia to detect diabetic retinopathy on IBM POWER8 + NVIDIA GPUs", available at: https://juliacomputing.com/blog/2016/11/16/deepeyes.html (accessed 15 July 2020).

Angell, B.J., Prinja, S., Gupt, A., Jha, V. and Jan, S. (2019), “The Ayushman Bharat Pradhan Mantri Jan Arogya Yojana and the path to universal health coverage in India: overcoming the challenges of stewardship and governance", PLoS Medicine, Vol. 16 No. 3, pp. 1-6.

Bakshi, H., Sharma, R. and Kumar, P. (2018), "Ayushman Bharat initiative: what we stand or gain to lose", Indian Journal of Community Medicine, Vol. 43 No. 2, pp. 63-66.

Chandwani, R.K. and Dwivedi, Y.K. (2015), "Telemedicine in India: current state, challenges and opportunities", Transforming Government: People, Process and Policy, Vol. 9 No. 4, pp. 393-400.

Dey, S. (2019), "Govt to use AI to curb frauds in Ayushman Bharat", The Times of India, 13 July, available at: https://timesofindia.indiatimes.com/india/govt-to-use-ai-to-curb-frauds-inayushman-bharat/articleshow/70200019.cms (accessed 28 March 2020).

Dhupar, V. (2017), "Deep Learning - the next big thing for the Indian government", Elets News Network, 15 September, available at: https://egov.eletsonline.com/2017/09/deep-learning-thenext-big-thing-for-the-indian-government/ (accessed 3 July 2020).

eHealth (2019), "Healthtech platform Pharmarack raises $\$ 3$ million in a series A funding", Elets News Network, 23 September, available at: https://ehealth.eletsonline.com/2019/09/healthtechplatform-pharmarack-raises-3-million-in-a-series-a-funding/ (accessed 3 July 2020).

Government of India (2018), “Ayushman Bharat - National Health Protection Mission”, available at: https://www.india.gov.in/spotlight/ayushman-bharat-national-health-protection-mission (accessed 19 March 2020).

Gupta, R. and Kumari, R. (2017), "Artificial Intelligence in public health: opportunities and challenges", JK Science, Vol. 19 No. 4, pp. 191-192.

IBM (2014), "Everyday ethics for Artificial Intelligence", available at: https://www.ibm.com/watson/ assets/duo/pdf/everydayethics.pdf (accessed 17 March 2020).

India Brand Equity Foundation (2019), "Healthcare industry in India", available at: https://www.ibef. org/industry/healthcare-india.aspx (accessed 15 March 2020).

Insilico (2020), "Homepage", available at: https://insilico.com (accessed 7 August 2020).

Jha, S.K. (2018), " 1 crore patients a year to be covered under Ayushman Bharat", Business World, 27 October, available at: http:/www.businessworld.in/article/-1-Crore-Patients-A-Year-To-BeCovered-Under-Ayushman-Bharat-/19-11-2018-164306/ (accessed 15 March 2020).

Jiang, F., et al. (2017), "Artificial Intelligence in healthcare: past, present and future", Stroke and Vascular Neurology, Vol. 2 No. 4, pp. 230-243.

Keshri, V. and Gupta, S. (2019), "Ayushman Bharat and road to universal health coverage in India", Journal of Mahatma Gandhi Institute of Medical Sciences, Vol. 24 No. 2, pp. 65-67. 
Lahariya, C. (2018), "Ayushman Bharat program and universal health coverage in India", Indian Pediatrics, Vol. 55 No. 6, pp. 495-506.

Lunit (2020), “Our Story”, available at: https://www.lunit.io/en/ (accessed 7 August 2020).

National Health Authority (2018), "Annual report 2018-19", available at: https://www.pmjay.gov.in/ sites/default/files/2019-09/Annual\%20Report $\% 20-\% 20 \mathrm{PMJAY} \% 20$ small\%20version_1.pdf (accessed 10 March 2020).

National Health Authority and Quality Council of India (2019), "Guidebook for AB PMJAY Quality Certification”, available at: https://www.pmjay.gov.in/sites/default/files/2019-09/Quality\% 20Certification\%20Process\%20Guidebook.pdf (accessed 15 March 2020).

NITI Aayog (2018), "National strategy for artificial intelligence", Discussion Paper, available at: https:// niti.gov.in/writereaddata/files/document_publication/NationalStrategy-for-AI-Discussion-Paper. pdf (accessed 12 February 2020).

PathAI (2020), "What we do", available at: https:/www.pathai.com (accessed 7 August 2020).

PwC (2018), "Reimagining the possible in the Indian healthcare ecosystem with emerging technologies", available at: https://www.pwc.in/assets/pdfs/publications/2018/reimagining-the-possible-in-theindian-healthcare-ecosystem-with-emerging-technologies.pdf (accessed 17 March 2020).

Radick, L.E. (2017), "Artificial Intelligence in healthcare: the current, compelling wave of interest", Healthcare Executive, Vol. 32 No. 5, pp. 20-22, 24-28.

Reddy, C.K., Aggarwal, C.C. and Acampora, G. (2015), Healthcare Data Analytics, CRC Press, Florida.

Reuters (2010), "Global healthcare fraud costs put at $\$ 260$ billion”, available at: https://www.reuters. com/article/us-healthcare-fraud/global-healthcare-fraud-costs-put-at-260-billion-idUSTRE60H0 1620100118 (accessed 17 March 2020).

Sense.ly (2020), “About us”, available at: https://www.sensely.com/about/ (accessed 7 August 2020).

Singh, P.K. (2018), "Ayushman Bharat: an ambitious set of reforms that should benefit millions of India's poor and vulnerable", World Health Organization, 1 October, available at: https://www. who.int/southeastasia/news/opinion-editorials/detail/ayushman-bharat-an-ambitious-set-ofreforms-that-should-benefit-millions-of-india-s-poor-and-vulnerable (accessed 21 March 2020).

Srivastava, S.K. (2018), “Artificial Intelligence: way forward for India”, Journal of Information Systems and Technology Management, Vol. 15, pp. 1-23.

Stead, W. (2018), "Clinical implications and challenges of Artificial Intelligence and Deep Learning", Journal of the American Medical Association, Vol. 320 No. 11, pp. 1107-1108.

Vadakkepat, P., Garg, H.K., Loh, A.P. and Tham, M.P. (2015), "Inclusive innovation: getting more from less for more", Journal of Frugal Innovation, Vol. 1 No. 1, pp. 1-2.

Westgate, A. (2017), "Health IT forecast", Managed Healthcare Executive, Vol. 27 No. 5, pp. 32-33.

\section{About the authors}

Raul V. Rodriguez is the Dean, School of Business, Woxsen University, Hyderabad, India. He is a registered expert in the fields of Artificial Intelligence, Intelligent Systems, Multi-agent Systems at the European Commission. He has been nominated for the "Forbes 30 Under 30 Europe" 2020 list. Raul V. Rodriguez is the corresponding author and can be contacted at: raulviro@gmail.com

Sanjivni Sinha, BS (North Dakota), MS (Minot State), MBA (Cardiff Metropolitan), is a Healthcare Business Analyst-Consultant at Citius Tech, Mumbai, India. While pursuing her undergraduate degree, she was a Research Assistant for a research project under the Environmental Department of North Dakota State University in the USA.

Sakshi Tripathi, BCom (A.K.S.), MBA (Cardiff Metropolitan), specialised in the fields of technology and fintech, is an Associate at HDFC Bank, Mumbai, India.

For instructions on how to order reprints of this article, please visit our website:

www.emeraldgrouppublishing.com/licensing/reprints.htm

Or contact us for further details: permissions@emeraldinsight.com
Impact of Artificial Intelligence in India 